\title{
A Técnica de Duplicação de Orifício na Plastia Valvar Mitral: 35 Anos de História
}

\author{
The Double-Orifice Technique in Mitral Valve Repair: 35 Years of History \\ Pablo Maria Alberto Pomerantzeff, ${ }^{1}$ C Carlos Manuel de Almeida Brandão, ${ }^{1}$ Arlindo Riso, ${ }^{1}$ Fabio Biscegli Jatene ${ }^{1}$ \\ Instituto do Coração do Hospital das Clínicas da Faculdade de Medicina da Universidade de São Paulo, ${ }^{1}$ São Paulo, SP - Brasil
}

As vantagens da plastia valvar mitral são menor morbimortalidade, redução do risco de tromboembolismo e endocardite, melhora na sobrevida e melhor preservação da função ventricular esquerda. A causa mais comum de regurgitação mitral degenerativa é a válvula mixomatosa com prolapso segmentar do folheto posterior; esta lesão pode ser corrigida por técnicas clássicas, como a ressecção quadrangular, mas outras lesões podem exigir técnicas cirúrgicas mais complexas, por exemplo, correção de prolapso do folheto anterior ou doença de Barlow. A plastia valvar mitral em pacientes reumáticos ainda é um desafio.

Em dezembro 1984, Adib Domingos Jatene MD, PhD realizou a primeira "duplicação de orifício mitral", em uma paciente feminina com insuficiência mitral por ruptura das cordas do folheto anterior (Figura 1). A técnica restaurou a competência mitral ancorando a borda livre dos folhetos em prolapso à borda livre correspondente do folheto oposto com um ponto de polipropileno 5-0 reforçado com compressas. A paciente apresentou boa evolução imediata e tardia.

Em 1998, Maisano et al. ${ }^{1}$ publicaram a técnica de "duplicação de orifício mitral", conhecida como a técnica "borda a borda", que vinha sendo realizada desde 1991, segundo os autores. Subsequentemente, Alfieri et al. publicaram os resultados de médio prazo desta técnica única, ${ }^{2}$ mostrando a sua eficácia e durabilidade, e o mesmo grupo recentemente publicou os resultados de longo prazo (18 anos). ${ }^{3}$

Desde 1980, nós realizamos a plastia valvar mitral com diferentes técnicas, incluindo a "duplicação de orifício mitral", conforme publicado em 1994 pelo nosso grupo, ${ }^{4}$ com bons resultados a longo prazo (17 anos). ${ }^{5}$ Realizamos este tipo de plastia valvar mitral em pacientes com anel valvar largo no sentido de não haver risco de causar estenose. Evidentemente, a degeneração dos tecidos das cúspides deve ser avaliada no ato operatório, assim como as cordas tendinosas e os músculos papilares, mas é necessário lembrar que, corrigida a insuficiência mitral, o estresse das cúspides é fortemente reduzido. Outro detalhe importante em qualquer plastia valvar mitral é a análise da coaptação entre as cúspides anterior e posterior, por meio do teste de solução salina, injetada pela mitral no ventrículo esquerdo. Quando utilizamos a duplicação de orifício mitral, devemos verificar não apenas a coaptação no local da duplicação, que obviamente é boa, visto que existe uma sutura entre as cúspides, mas também a coaptação em toda a extensão da borda entre as cúspides anterior e posterior.

\section{Palavras-chave}

Valva Mitral/cirurgia; Doenças das Valvas Cardíacas/ cirurgia; Morbidade e Mortalidade; Tromboembolismo/ prevenção e controle; Endocardite/prevenção e controle.

\section{Correspondência: Pablo Maria Alberto Pomerantzeff}

Instituto do Coração do Hospital das Clínicas da Faculdade de Medicina da

Universidade de São Paulo - Av. Doutor Enéas de Carvalho Aguiar, 44. CEP

05403000, São Paulo, SP - Brasil

E-mail: dcipablo@incor.usp.br, pablo.pomerantzeff@hotmail.com

Artigo recebido em 01/02/2021, revisado em 03/03/2021, aceito em 03/03/2021

DOI: https://doi.org/10.36660/abc.20210067 


\section{Editorial}
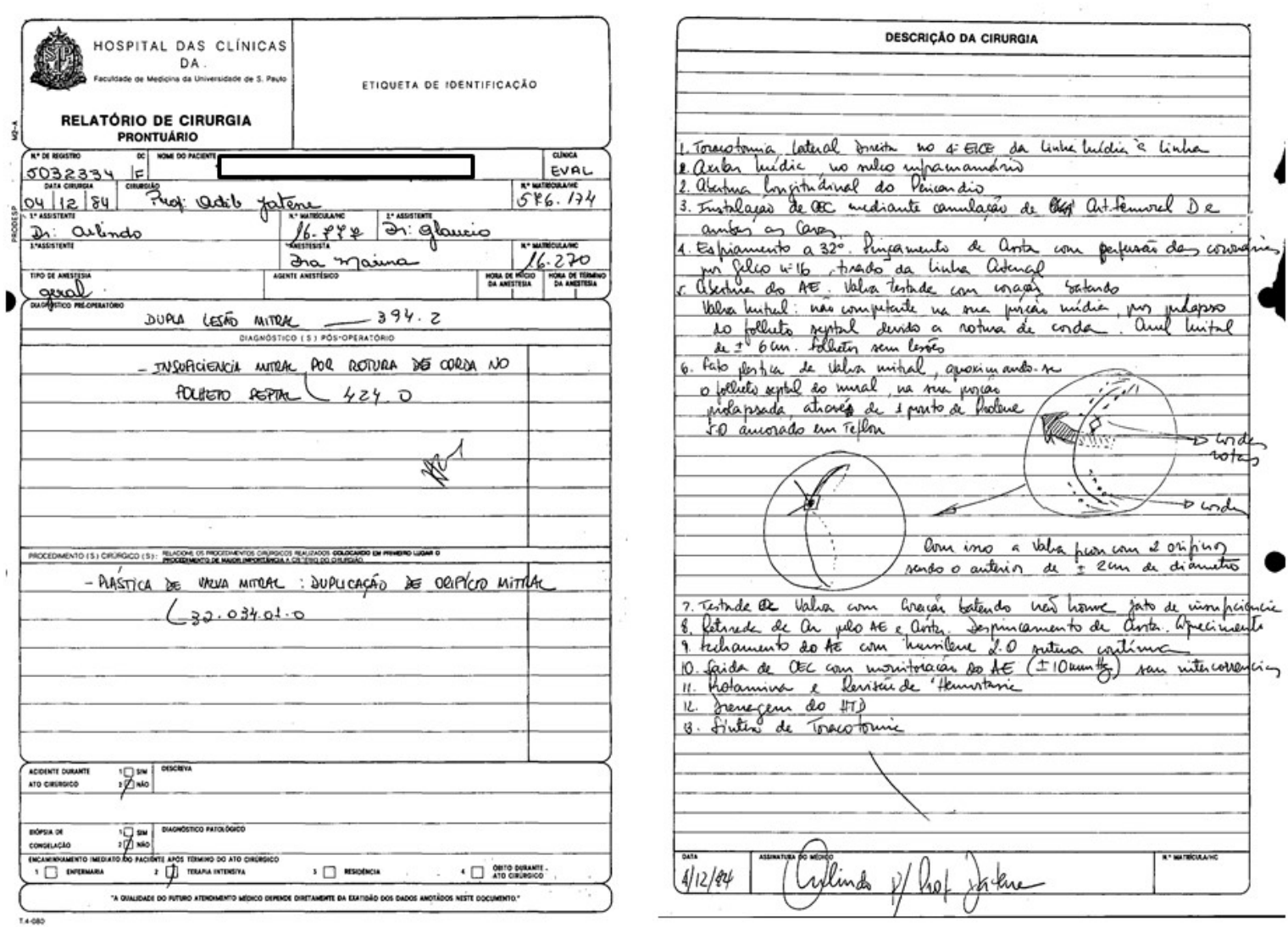

Figura 1 - Relatório de cirurgia de plastia valvar mitral pela técnica de duplicação de orificio.

\section{Referências}

1. Maisano F, Torraca L, Oppizzi M, Stefano PL, D’Addario G, La Canna G, et al. The edge-to-edge technique: a simplified method to correct mitral insufficiency. Eur J Cardio-thorac Surg. 1998;13(3):240-6.

2. Alfieri O, Maisano F, De Bonis M, Stefano PL, Torraca L, Oppizzi M. The double-orifice technique in mitral valve repair: A simple solution for complex problems. J Thorac Cardiovasc Surg. 2001; 122(4):674-81.

3. De Bonis M, Lapenna E, Maisano, F, Barili F, La Canna G, Buzzati N, et al. Long-Term Results ( $\leq 18$ Years) of the Edge-to-Edge Mitral Valve Repair
Without Annuloplasty in Degenerative Mitral Regurgitation: Implications for the Percutaneous Approach. Circulation. 2014;130(11 Suppl 1):S19-24.

4. Pomerantzeff PMA, Brandão CMA, Monteiro ACM, Nercessian AC, Zeratti $A E$, Stolf NAG, et al. Mitral valve repair: 12 years experience and evolution of techniques. Braz J Cardiovasc Surg. 1994; 9(1):22-8.

5. Pomerantzeff PMA, Brandão CMA, Faber CN, Fonseca MH, Puig LB, Grinberg M, et al. Mitral valve repair: seventeen years experience. Braz J Cardiovasc Surg. 1999;14(3):185-90. 